\title{
Robust Adaptive Finite-Time Synchronization of Two Different Chaotic Systems with Parameter Uncertainties
}

\author{
Yun-An Hu, Hai-Yan Li, Chun-Ping Zhang, and Liang Liu \\ Department of Control Engineering, Naval Aeronautical and Astronautical University, Yantai, \\ Shandong 264001, China \\ Correspondence should be addressed to Yun-An Hu, hya507@sina.com
}

Received 10 April 2012; Revised 20 July 2012; Accepted 22 July 2012

Academic Editor: Laurent Gosse

Copyright (C) 2012 Yun-An Hu et al. This is an open access article distributed under the Creative Commons Attribution License, which permits unrestricted use, distribution, and reproduction in any medium, provided the original work is properly cited.

\begin{abstract}
This paper is concerned with the finite-time synchronization problem for two different chaotic systems with parameter uncertainties. Using finite-time control approach and robust control method, an adaptive synchronization scheme is proposed to make the synchronization errors of the systems with parameter uncertainties zero in a finite time. On the basis of Lyapunov stability theory, appropriate adaptive laws are derived to deal with the unknown parameters of the systems. And the convergence of the parameter errors is guaranteed in a finite time. The proposed method can be applied to a variety of chaos systems. Numerical simulations are given to demonstrate the efficiency of the proposed control scheme.
\end{abstract}

\section{Introduction}

In the past few decades, chaos synchronization has gained much attention from various fields [1-3], since Pecora and carroll [4] introduced a method to synchronize two identical chaotic systems with different initial conditions in 1990. Most of the works on chaos synchronization have focused on two identical chaotic systems [5-11]. However, in many real world applications, there are no exactly two identical chaotic systems. Therefore, the problem of chaos synchronization between two different chaotic systems with uncertainties is an important research issue [12]. Different synchronization control methods for two different chaotic systems, such as adaptive control [13-21], nonlinear feedback control [22], backstepping [23, 24], fuzzy technique [25-27], and sliding mode control [28-30], have been proposed to solve the synchronization problem.

Since some systems' parameters cannot be exactly known in advance, many efforts have been devoted to adaptive synchronization. In [18, 31], Huang discussed the 
synchronizations between Lorenz-Stenflo (LS) system and CYQY system, and between LS system and hyperchaotic Chen system with fully uncertain parameters. Wang et al. [15] designed a general adaptive robust controller and parameter update laws which made the drive-response systems with different structures asymptotically synchronized. In [16], the sufficient conditions for achieving synchronization between generalized Henon-Heiles system and hyperchaotic Chen system with unknown parameters were derived based on Lyapunov stability theory. A new adaptive synchronization scheme by pragmatical asymptotically stability theorem was proposed for two different uncertain chaotic systems [17], but the unknown signals were used in the controller. Chaos synchronization between two different chaotic systems with uncertainties in both master and slave chaotic systems remains a challenging problem [30].

Most methods only guarantee the asymptotic stability of the synchronization error dynamics, namely, the trajectories of the slave system approach the trajectories of the master system as $t \rightarrow \infty$. From a practical point of view, however, it is more valuable that the synchronization objective is realized in a finite time [28]. In recent years, some researchers have applied finite-time control techniques, such as nonsingular terminal sliding mode control method [32], CLF-based method [33, 34], sliding mode control method [28-30], and the finite-time stability theory-based method $[28,35,36]$, to realize synchronization.

Compared with the existing results in the literature, there are three advantages which make our approach attractive. First, based on the finite-time control technique, adaptive control, and robust control, a new synchronization method is presented for a wide class of nonlinear systems. Second, it guarantees that all the errors are driven to zero in a finite time even for the systems with parameter uncertainties. Third, it guarantees that all the parameter errors converge to zero in a finite time.

In this paper, an adaptive finite-time synchronization scheme is proposed for a class of chaotic systems. The rest of the paper is organized as follows. In Section 2, we introduce the chaotic systems considered in this paper and preliminary lemmas. In Section 3, the proposed finite-time controller is designed to synchronize two different chaotic systems. We give the simulation results and the conclusions in Sections 4 and 5, respectively.

\section{System Description}

Consider the following master chaotic system:

$$
\dot{x}=\left(A_{1}+\Delta A_{1}\right) x+\left(B_{1}+\Delta B_{1}\right) f_{1}(x),
$$

where $x=\left[x_{1}, x_{2}, \ldots, x_{n}\right]^{T} \in R^{n}$ denotes a state vector, $f_{1}$ is a nonlinear continuous vector function, $A_{1}$ and $B_{1}$ are $n \times n$ nominal coefficient matrices, $\Delta A_{1}$ and $\Delta B_{1}$ are unknown parts of $n \times n$ coefficient matrices.

The slave system is given with

$$
\dot{y}=\left(A_{2}+\Delta A_{2}\right) y+\left(B_{2}+\Delta B_{2}\right) f_{2}(y)+u,
$$

where $y=\left[y_{1}, y_{2}, \ldots, y_{n}\right]^{T} \in R^{n}$ denotes a state vector, $f_{2}$ is a nonlinear continuous vector function, $A_{2}$ and $B_{2}$ are $n \times n$ nominal coefficient matrices, $\Delta A_{2}$ and $\Delta B_{2}$ are unknown parts 
of $n \times n$ coefficient matrices, and $u=\left[u_{1}(t), u_{2}(t), \ldots, u_{n}(t)\right]^{T} \in R^{n}$ is a control input vector to be designed.

Subtracting (2.1) from (2.2) yields the error dynamical system as follows:

$$
\dot{e}=\left(A_{2}+\Delta A_{2}\right) y+\left(B_{2}+\Delta B_{2}\right) f_{2}(y)-\left(A_{1}+\Delta A_{1}\right) x-\left(B_{1}+\Delta B_{1}\right) f_{1}(x)+u,
$$

where $e=y-x$. Note that only a part of elements of the coefficient matrices unknown, without loss of generality, we assume that the number of the unknown elements of the $i$ th row of $\Delta A_{1}$ is $N_{A 1 i}$, that of $\Delta A_{2}$ is $N_{A 2 i}$, that of $\Delta B_{1}$ is $N_{B 1 i}$, and that of $\Delta B_{2}$ is $N_{B 2 i}$. Then (2.3) can be rewritten as

$$
\begin{aligned}
\dot{e}= & A_{2} y+B_{2} f_{2}(y)-A_{1} x-B_{1} f_{1}(x)+u \\
& +\left[\begin{array}{c}
\sum_{i=1}^{N_{A 21}} \delta a_{21 i} \bar{y}_{1 i} \\
\vdots \\
\sum_{i=1}^{N_{A 2 n}} \delta a_{2 n i} \bar{y}_{n i}
\end{array}\right]+\left[\begin{array}{c}
\sum_{i=1}^{N_{B 21}} \delta b_{21 i} \bar{f}_{21 i} \\
\vdots \\
\sum_{i=1}^{N_{B 2 n}} \delta b_{2 n i} \bar{f}_{2 n i}
\end{array}\right]-\left[\begin{array}{c}
\sum_{i=1}^{N_{A 11}} \delta a_{11 i} \bar{x}_{1 i} \\
\vdots \\
\sum_{i=1}^{N_{A 1 n}} \delta a_{1 n i} \bar{x}_{n i}
\end{array}\right]-\left[\begin{array}{c}
\sum_{i=1}^{N_{B 11}} \delta b_{11 i} \bar{f}_{11 i} \\
\vdots \\
\sum_{i=1}^{N_{B 1 n}} \delta b_{1 n i} \bar{f}_{1 n i}
\end{array}\right],
\end{aligned}
$$

where $\delta a_{* j i}$ are nonzero elements of the $j$ th row of $\Delta A_{*}, \bar{y}_{j i}$ are corresponding elements of $y$, $\delta b_{* j i}$ are nonzero elements of the $j$ th row of $\Delta B_{*}$ and $\bar{f}_{* j i}$ are corresponding elements of $f_{* \prime}$ $j=1, \ldots, n$.

Assumption 2.1. The unknown parameters are norm-bounded, that is,

$$
\left|\delta a_{* j i}\right| \leq d_{a_{*} j i}, \quad\left|\delta b_{* j i}\right| \leq d_{b_{*} j i}
$$

where $d_{a_{*} j i}$ and $d_{b_{* j i}}$ are known positive constants.

Definition 2.2 (see [28]). Consider the master and slave chaotic systems described by (2.1) and (2.2), respectively. If there exists a constant $T=T(e(0))>0$, such that

$$
\lim _{t \rightarrow T}\|e(t)\|=0
$$

and $\|e(t)\| \equiv 0$, if $t \geq T$, then the chaos synchronization between the systems (2.1) and (2.2) is achieved in a finite time.

Lemma 2.3 (see [28]). Consider the system

$$
\dot{x}=f(x), \quad f(0)=0, \quad x \in R^{n},
$$

where $f: D \rightarrow R^{n}$ is continuous on an open neighborhood $D \in R^{n}$. 
Suppose there exists a continuous differential positive-definite function $V(x): D \rightarrow$ $R$, real numbers $p>0,0<\eta<1$, such that

$$
\dot{V}(x)+p V^{\eta}(x) \leq 0, \quad \forall x \in D .
$$

Then, the origin of system (2.7) is a locally finite-time stable equilibrium, and the settling time, depending on the initial state $\mathrm{x}(0)=\mathrm{x}_{0}$, satisfies

$$
T\left(x_{0}\right) \leq \frac{V^{1-\eta}\left(x_{0}\right)}{p(1-\eta)} .
$$

In addition, if $D=R^{n}$ and $V(x)$ is also radially unbounded (i.e., $V(x) \rightarrow+\infty$ as $\|x\| \rightarrow+\infty)$, then the origin is a globally finite-time stable equilibrium of system (2.7).

Lemma 2.4 (see [28]). Suppose $a_{1}, a_{2}, \ldots, a_{n}$, and $0<q<2$ are all real numbers, then the following inequality holds:

$$
\left|a_{1}\right|^{q}+\left|a_{2}\right|^{q}+\cdots+\left|a_{n}\right|^{q} \geq\left(a_{1}^{2}+a_{2}^{2}+\cdots+a_{n}^{2}\right)^{q / 2} .
$$

\section{Synchronization of Two Different Chaotic Systems with Parameter Uncertainties}

Consider two different chaotic systems (2.1) and (2.2) from different initial states. The aim of controller design is to determine appropriate $u$ such that

$$
\lim _{t \rightarrow T} e=0
$$

Now we are ready to give the design steps.

Define Lyapunov function

$$
\begin{aligned}
V=\frac{1}{2} e^{T} e & +\frac{1}{2} \sum_{j=1}^{n} \sum_{i=1}^{N_{A 2 j}}\left(1+k_{d j}\right)^{-1} \gamma_{a 2 j i}^{-1} \delta \tilde{a}_{2 j i}^{2}+\frac{1}{2} \sum_{j=1}^{n} \sum_{i=1}^{N_{A 1 j}}\left(1+k_{d j}\right)^{-1} \gamma_{a 1 j i}^{-1} \delta \tilde{a}_{1 j i}^{2} \\
& +\frac{1}{2} \sum_{j=1}^{n} \sum_{i=1}^{N_{B 2 j}}\left(1+k_{d j}\right)^{-1} \gamma_{b 2 j i}^{-1} \delta \tilde{b}_{2 j i}^{2}+\frac{1}{2} \sum_{j=1}^{n} \sum_{i=1}^{N_{B 1 j}}\left(1+k_{d j}\right)^{-1} \gamma_{b 1 j i}^{-1} \delta \tilde{b}_{1 j i}^{2},
\end{aligned}
$$

where $\delta \widetilde{a}_{2 j i}=\delta \widehat{a}_{2 j i}-\delta a_{2 j i}, \delta \widetilde{a}_{1 j i}=\delta \widehat{a}_{1 j i}-\delta a_{1 j i}, \delta \tilde{b}_{2 j i}=\delta \widehat{b}_{2 j i}-\delta b_{2 j i}, \delta \tilde{b}_{1 j i}=\delta \widehat{b}_{1 j i}-\delta b_{1 j i}$, and $\delta \widehat{a}_{2 j i}, \delta \widehat{a}_{1 j i}, \delta \widehat{b}_{2 j i}, \delta \widehat{b}_{1 j i}$ are estimation values of $\delta a_{2 j i}, \delta a_{1 j i}, \delta b_{2 j i}, \delta b_{1 j i}$, respectively, and $\gamma_{a 2 j i}^{-1}$, $r_{a 1 j i}^{-1}, \gamma_{b 2 j i}^{-1}, \gamma_{b 1 j i}^{-1}, k_{d j}$ are constants greater than zero. 
Journal of Applied Mathematics

Taking the time derivative of (3.2) gives

$$
\begin{aligned}
\dot{V}=e^{T} \dot{e} & +\sum_{j=1}^{n} \sum_{i=1}^{N_{A 2 j}}\left(1+k_{d j}\right)^{-1} \gamma_{a 2 j i}^{-1} \delta \tilde{a}_{2 j i} \delta \dot{\hat{a}}_{2 j i}+\sum_{j=1}^{n} \sum_{i=1}^{N_{A 1 j}}\left(1+k_{d j}\right)^{-1} \gamma_{a 1 j i}^{-1} \delta \tilde{a}_{1 j i} \delta \dot{\vec{a}}_{1 j i} \\
& +\sum_{j=1}^{n} \sum_{i=1}^{N_{B 2 j}}\left(1+k_{d j}\right)^{-1} \gamma_{b 2 j i}^{-1} \delta \tilde{b}_{2 j i} \delta \hat{\vec{b}}_{2 j i}+\sum_{j=1}^{n} \sum_{i=1}^{N_{B 1 j}}\left(1+k_{d j}\right)^{-1} \gamma_{b 1 j i}^{-1} \delta \tilde{b}_{1 j i} \delta \dot{\vec{b}}_{1 j i} .
\end{aligned}
$$

Design the control law as

$$
\begin{aligned}
u= & -K e-K_{D} \dot{e}+\alpha_{s}-\left[c_{1} \operatorname{sgn}\left(e_{1}\right)\left|e_{1}\right|^{\alpha}, \ldots, c_{n} \operatorname{sgn}\left(e_{n}\right)\left|e_{n}\right|^{\alpha}\right]^{T} \\
& -\mu\left[\sum_{j=1}^{n} \sum_{i=1}^{N_{A 2 j}}\left(\left|\delta \widehat{a}_{2 j i}\right|+d_{a 2 j i}\right)^{1+\alpha}+\sum_{j=1}^{n} \sum_{i=1}^{N_{A 1 j}}\left(\left|\delta \widehat{a}_{1 j i}\right|+d_{a 1 j i}\right)^{1+\alpha}\right. \\
& \left.+\sum_{j=1}^{n} \sum_{i=1}^{N_{B 2 j}}\left(\left|\delta \widehat{b}_{2 j i}\right|+d_{b 2 j i}\right)^{1+\alpha}+\sum_{j=1}^{n} \sum_{i=1}^{N_{B 1 j}}\left(\left|\delta \widehat{b}_{1 j i}\right|+d_{b 1 j i}\right)^{1+\alpha}\right] \alpha_{e} \\
& +\left[\sum_{i=1}^{N_{A 11}} \delta \widehat{a}_{11 i} \bar{y}_{1 i}, \ldots, \sum_{i=1}^{N_{A 1 n}} \delta \widehat{a}_{1 n i} \bar{y}_{n i}\right]^{T}-\left[\sum_{i=1}^{N_{B 21}} \delta \widehat{b}_{21 i} \bar{f}_{21 i}, \ldots, \sum_{i=1}^{N_{A 1 n}} \delta \widehat{b}_{2 n i} \bar{f}_{2 n i}\right]^{T} \\
& -\left[\sum_{i=1}^{N_{A 21}} \delta \widehat{a}_{21 i} \bar{y}_{1 i}, \ldots, \sum_{i=1}^{N_{A 2 n}} \delta \widehat{a}_{2 n i} \bar{y}_{n i}\right]^{T}+\left[\sum_{i=1}^{N_{B 11}} \delta \widehat{b}_{11 i} \bar{f}_{11 i}, \ldots, \sum_{i=1}^{N_{B 1 n}} \delta \widehat{b}_{1 n i} \bar{f}_{1 n i}\right]^{T},
\end{aligned}
$$

where $K=\operatorname{diag}\left\{k_{1}, k_{2}, \ldots, k_{n}\right\}, k_{i}>0, K_{D}=\operatorname{diag}\left\{k_{d 1}, k_{d 2}, \ldots, k_{d n}\right\}, k_{d i}>0$, and $c_{i}>0$ are

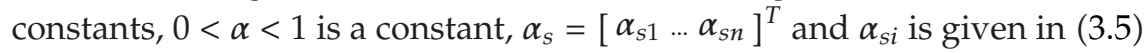

$$
\alpha_{s i}= \begin{cases}-\frac{\left(\partial V_{A}\right) /\left(\partial e_{i}\right) f_{A i}+K_{A i} \sqrt{\left(\left(\partial V_{A}\right) /\left(\partial e_{i}\right) f_{A i}\right)^{2}+\left(\left(\partial V_{A}\right) /\left(\partial e_{i}\right)\right)^{4}}}{\left(\partial V_{A}\right) /\left(\partial e_{i}\right)}, & \text { if } \frac{\partial V_{A}}{\partial e_{i}} \neq 0 \\ 0, \text { if } \frac{\partial V_{A}}{\partial e_{i}}=0, & i=1, \ldots, n,\end{cases}
$$

where $V_{A}=(1 / 2) e^{T} e, f_{A}=-A_{2} y-B_{2} f_{2}(y)+A_{1} x+B_{1} f_{1}(x)$ and $K_{A i}>0$. And $\alpha_{e}=\left[\alpha_{e 1} \ldots \alpha_{e n}\right]^{T}$ and $\alpha_{e i}$ is given in

$$
\alpha_{e i}= \begin{cases}\frac{1}{e_{i}}, & \text { if }\left|e_{i}\right| \geq e_{\sigma} \\ \frac{\operatorname{sgn}\left(e_{i}\right)}{e_{\sigma}}, & \text { if }\left|e_{i}\right|<e_{\sigma}\end{cases}
$$

where $e_{\sigma}>0$ is a small constant. 
Substituting (3.4) into (2.4) gives

$$
\begin{aligned}
& \dot{e}=-K\left(I+K_{D}\right)^{-1} e-\left[\begin{array}{c}
\sum_{i=1}^{N_{A 21}}\left(1+k_{d 1}\right)^{-1} \delta \tilde{a}_{21 i} \bar{y}_{1 i} \\
\vdots \\
\sum_{i=1}^{N_{A 2 n}}\left(1+k_{d n}\right)^{-1} \delta \tilde{a}_{2 n i} \bar{y}_{n i}
\end{array}\right]-\left[\begin{array}{c}
\sum_{i=1}^{N_{B 21}}\left(1+k_{d 1}\right)^{-1} \delta \tilde{b}_{21 i} \bar{f}_{21 i} \\
\vdots \\
\sum_{i=1}^{N_{B 2 n}}\left(1+k_{d n}\right)^{-1} \delta \tilde{b}_{2 n i} \bar{f}_{2 n i}
\end{array}\right] \\
& +f_{A}+\alpha_{S}+\left[\begin{array}{c}
\sum_{i=1}^{N_{A 11}}\left(1+k_{d 1}\right)^{-1} \delta \tilde{a}_{11 i} \bar{x}_{1 i} \\
\vdots \\
\sum_{i=1}^{N_{A 1 n}}\left(1+k_{d n}\right)^{-1} \delta \tilde{a}_{1 n i} \bar{x}_{n i}
\end{array}\right]+\left[\begin{array}{c}
\sum_{i=1}^{N_{B 11}}\left(1+k_{d 1}\right)^{-1} \delta \tilde{b}_{11 i} \bar{f}_{11 i} \\
\vdots \\
\sum_{i=1}^{N_{B 1 n}}\left(1+k_{d n}\right)^{-1} \delta \tilde{b}_{1 n i} \bar{f}_{1 n i}
\end{array}\right] \\
& -\mu\left(I+K_{D}\right)^{-1}\left[\sum_{j=1}^{n} \sum_{i=1}^{N_{A 2 j}}\left(\left|\delta \widehat{a}_{2 j i}\right|+d_{a 2 j i}\right)^{1+\alpha}+\sum_{j=1}^{n} \sum_{i=1}^{N_{A 1 j}}\left(\left|\delta \widehat{a}_{1 j i}\right|+d_{a 1 j i}\right)^{1+\alpha}\right. \\
& \left.+\sum_{j=1}^{n} \sum_{i=1}^{N_{B 2 j}}\left(\left|\delta \widehat{b}_{2 j i}\right|+d_{b 2 j i}\right)^{1+\alpha}+\sum_{j=1}^{n} \sum_{i=1}^{N_{B 1 j}}\left(\left|\delta \widehat{b}_{1 j i}\right|+d_{b 1 j i}\right)^{1+\alpha}\right] \alpha_{e}
\end{aligned}
$$

$-\left(I+K_{D}\right)^{-1}\left[c_{1} \operatorname{sgn}\left(e_{1}\right)\left|e_{1}\right|^{\alpha}, \ldots, c_{n} \operatorname{sgn}\left(e_{n}\right)\left|e_{n}\right|^{\alpha}\right]^{T}$.

Case $1\left(\left|\mathrm{e}_{\mathrm{i}}\right| \geq \mathrm{e}_{\sigma}\right)$. Substituting (3.7) into (3.3) yields

$$
\begin{gathered}
\dot{V} \leq-e^{T} K\left(I+K_{D}\right)^{-1} e-\sum_{i=1}^{n} c_{i}\left(1+k_{d i}\right)^{-1}\left|e_{i}\right|^{1+\alpha} \\
-\mu k_{d}\left[\sum_{j=1}^{n} \sum_{i=1}^{N_{A 2 j}}\left(\left|\delta \widehat{a}_{2 j i}\right|+d_{a 2 j i}\right)^{1+\alpha}+\sum_{j=1}^{n} \sum_{i=1}^{N_{A 1 j}}\left(\left|\delta \widehat{a}_{1 j i}\right|+d_{a 1 j i}\right)^{1+\alpha}\right. \\
\left.+\sum_{j=1}^{n} \sum_{i=1}^{N_{B 2 j}}\left(\left|\delta \widehat{b}_{2 j i}\right|+d_{b 2 j i}\right)^{1+\alpha}+\sum_{j=1}^{n} \sum_{i=1}^{N_{B 1 j}}\left(\left|\delta \widehat{b}_{1 j i}\right|+d_{b 1 j i}\right)^{1+\alpha}\right] \\
-\sum_{j=1}^{n} e_{j} \sum_{i=1}^{N_{A 2 j}}\left(1+k_{d j}\right)^{-1} \delta \tilde{a}_{2 j i} \bar{y}_{j i}+\sum_{j=1}^{n} e_{j} \sum_{i=1}^{N_{A 1 j}}\left(1+k_{d j}\right)^{-1} \delta \tilde{a}_{1 j i} \bar{x}_{j i} \\
-\sum_{j=1}^{n} e_{j} \sum_{i=1}^{N_{B 2 j}}\left(1+k_{d j}\right)^{-1} \delta \tilde{b}_{2 j i} \bar{f}_{2 j i}+\sum_{j=1}^{n} e_{j} \sum_{i=1}^{N_{B 1 j}}\left(1+k_{d j}\right)^{-1} \delta \tilde{b}_{1 j i} \bar{f}_{1 j i}
\end{gathered}
$$


Journal of Applied Mathematics

$$
\begin{aligned}
& +\sum_{j=1}^{n} \sum_{i=1}^{N_{A 2 j}}\left(1+k_{d j}\right)^{-1} \gamma_{a 2 j i}^{-1} \delta \tilde{a}_{2 j i} \delta \dot{a}_{2 j i}+\sum_{j=1}^{n} \sum_{i=1}^{N_{A 1 j}}\left(1+k_{d j}\right)^{-1} \gamma_{a 1 j i}^{-1} \delta \tilde{a}_{1 j i} \delta \dot{a}_{1 j i} \\
& +\sum_{j=1}^{n} \sum_{i=1}^{N_{B 2 j}}\left(1+k_{d j}\right)^{-1} \gamma_{b 2 j i}^{-1} \delta \tilde{b}_{2 j i} \delta \dot{\hat{b}}_{2 j i}+\sum_{j=1}^{n} \sum_{i=1}^{N_{B 1 j}}\left(1+k_{d j}\right)^{-1} \gamma_{b 1 j i}^{-1} \delta \tilde{b}_{1 j i} \delta \dot{\vec{b}}_{1 j i}
\end{aligned}
$$

where $k_{d}=\min \left\{\left(1+k_{d 1}\right)^{-1},\left(1+k_{d 2}\right)^{-1}, \ldots,\left(1+k_{d n}\right)^{-1}\right\}$. Choosing the updating law as

$$
\begin{aligned}
\delta \dot{\vec{a}}_{2 j i} & = \begin{cases}\gamma_{a 2 j i} e_{j} \bar{y}_{j i}, & \text { if }\left|\widehat{a}_{2 j i}\right|<d_{a 2 j i} \\
0, & \text { otherwise, }\end{cases} \\
\delta \dot{\vec{a}}_{1 j i} & = \begin{cases}-\gamma_{a 1 j i} e_{j} \bar{x}_{j i}, & \text { if }\left|\widehat{a}_{1 j i}\right|<d_{a 1 j i} \\
0, & \text { otherwise, }\end{cases} \\
\delta \dot{\hat{b}}_{2 j i} & = \begin{cases}\gamma_{b 2 j i} e_{j} \bar{f}_{2 j i}, & \text { if }\left|\widehat{b}_{2 j i}\right|<d_{b 2 j i} \\
0, & \text { otherwise, }\end{cases} \\
\delta \dot{\hat{b}}_{1 j i} & = \begin{cases}-\gamma_{b 1 j i} e_{j} \bar{f}_{1 j i}, & \text { if }\left|\widehat{b}_{1 j i}\right|<d_{b 1 j i} \\
0, & \text { otherwise. }\end{cases}
\end{aligned}
$$

Substituting (3.9) into (3.8) yields

$$
\begin{aligned}
\dot{V} \leq & -e^{T} K\left(I+K_{D}\right)^{-1} e-\sum_{i=1}^{n} c_{i}\left(1+k_{d i}\right)^{-1}\left|e_{i}\right|^{1+\alpha} \\
-\mu k_{d} & {\left[\sum_{j=1}^{n} \sum_{i=1}^{N_{A 2 j}}\left(\left|\delta \widehat{a}_{2 j i}\right|+d_{a 2 j i}\right)^{1+\alpha}+\sum_{j=1}^{n} \sum_{i=1}^{N_{A 1 j}}\left(\left|\delta \widehat{a}_{1 j i}\right|+d_{a 1 j i}\right)^{1+\alpha}\right.} \\
& \left.+\sum_{j=1}^{n} \sum_{i=1}^{N_{B 2 j}}\left(\left|\delta \widehat{b}_{2 j i}\right|+d_{b 2 j i}\right)^{1+\alpha}+\sum_{j=1}^{n} \sum_{i=1}^{N_{B 1 j}}\left(\left|\delta \widehat{b}_{1 j i}\right|+d_{b 1 j i}\right)^{1+\alpha}\right] .
\end{aligned}
$$

Since

$$
\begin{aligned}
& \left|\delta \widehat{a}_{2 j i}-\delta a_{2 j i}\right| \leq\left|\delta \widehat{a}_{2 j i}\right|+\left|\delta a_{2 j i}\right| \leq\left|\delta \widehat{a}_{2 j i}\right|+d_{a 2 j i}, \\
& \left|\delta \widehat{a}_{1 j i}-\delta a_{1 j i}\right| \leq\left|\delta \widehat{a}_{1 j i}\right|+\left|\delta a_{1 j i}\right| \leq\left|\delta \widehat{a}_{1 j i}\right|+d_{a 1 j i}, \\
& \left|\delta \widehat{b}_{2 j i}-\delta b_{2 j i}\right| \leq\left|\delta \widehat{b}_{2 j i}\right|+\left|\delta b_{2 j i}\right| \leq\left|\delta \widehat{b}_{2 j i}\right|+d_{b 2 j i}, \\
& \left|\delta \widehat{b}_{1 j i}-\delta b_{1 j i}\right| \leq\left|\delta \widehat{b}_{1 j i}\right|+\left|\delta b_{1 j i}\right| \leq\left|\delta \widehat{b}_{1 j i}\right|+d_{b 1 j i}
\end{aligned}
$$


hold, one can conclude that $-\left(\left|\delta \widehat{a}_{2 j i}\right|+d_{a 2 j i}\right)^{1+\alpha} \leq-\left|\delta \widehat{a}_{2 j i}-\delta a_{2 j i}\right|^{1+\alpha}$,

$$
\begin{aligned}
& -\left(\left|\delta \widehat{a}_{1 j i}\right|+d_{a 1 j i}\right)^{1+\alpha} \leq-\left|\delta \widehat{a}_{1 j i}-\delta a_{1 j i}\right|^{1+\alpha}, \\
& -\left(\left|\delta \widehat{b}_{2 j i}\right|+d_{b 2 j i}\right)^{1+\alpha} \leq-\left|\delta \widehat{b}_{2 j i}-\delta b_{2 j i}\right|^{1+\alpha},
\end{aligned}
$$

and $-\left(\left|\delta \widehat{b}_{1 j i}\right|+d_{b 1 j i}\right)^{1+\alpha} \leq-\left|\delta \widehat{b}_{1 j i}-\delta b_{1 j i}\right|^{1+\alpha}$. Therefore, the inequality (3.10) can be rewritten as

$$
\begin{gathered}
\dot{V} \leq-e^{T} K\left(I+K_{D}\right)^{-1} e-\sum_{i=1}^{n} c_{i}\left(1+k_{d i}\right)^{-1}\left|e_{i}\right|^{1+\alpha} \\
-\mu k_{d}\left[\sum_{j=1}^{n} \sum_{i=1}^{N_{A 2 j}}\left|\delta \widehat{a}_{2 j i}-\delta a_{2 j i}\right|^{1+\alpha}+\sum_{j=1}^{n} \sum_{i=1}^{N_{A 1 j}}\left|\delta \widehat{a}_{1 j i}-\delta a_{1 j i}\right|^{1+\alpha}\right. \\
\left.+\sum_{j=1}^{n} \sum_{i=1}^{N_{B 2 j}}\left|\delta \widehat{b}_{2 j i}-\delta b_{2 j i}\right|^{1+\alpha}+\sum_{j=1}^{n} \sum_{i=1}^{N_{B 1 j}}\left|\delta \widehat{b}_{1 j i}-\delta b_{1 j i}\right|^{1+\alpha}\right] \\
\leq-c_{\mu}\left[\sum_{i=1}^{n}\left|e_{i}\right|^{2}+\sum_{j=1}^{n} \sum_{i=1}^{N_{A 2 j}}\left|\delta \widehat{a}_{2 j i}-\delta a_{2 j i}\right|^{2}+\sum_{j=1}^{n} \sum_{i=1}^{N_{A 1 j}}\left|\delta \widehat{a}_{1 j i}-\delta a_{1 j i}\right|^{2}\right. \\
\left.+\sum_{j=1}^{n} \sum_{i=1}^{N_{B 2 j}}\left|\delta \widehat{b}_{2 j i}-\delta b_{2 j i}\right|^{2}+\sum_{j=1}^{n} \sum_{i=1}^{N_{B 1 j}}\left|\delta \widehat{b}_{1 j i}-\delta b_{1 j i}\right|^{2}\right]^{(1+\alpha) / 2} \\
\leq-c_{\mu} V^{(1+\alpha) / 2},
\end{gathered}
$$

where $c_{\mu}=\min \left\{c_{i}\left(1+k_{d i}\right)^{-1}, k_{i}\left(1+k_{d i}\right)^{-1}, \mu k_{d}, i=1, \ldots, n\right\}$. According to Lemma 2.3, $e \rightarrow$ $B_{e_{\sigma}}$ in a finite time, where $B_{e_{\sigma}} \triangleq\left\{e \| e_{i} \mid \leq e_{\sigma}, i=1, \ldots, n\right\}$.

Case $2\left(\left|e_{\mathrm{i}}\right|<e_{\sigma}\right)$. Using (3.3)-(3.7) and (3.9), it is easy to show that

$$
\dot{V} \leq-e^{T} K\left(I+K_{D}\right)^{-1} e-\sum_{i=1}^{n} c_{i}\left(1+k_{d i}\right)^{-1}\left|e_{i}\right|^{1+\alpha}
$$

holds. According to Barbalat's lemma [37], we can conclude that $e \rightarrow 0$ as $t \rightarrow \infty$.

From the discussion above, we have the following result.

Theorem 3.1. For the systems (2.1) and (2.2), under Assumption 2.1, if the control law is designed as (3.4), updating laws are chosen as (3.9), then e will converge to $B_{e_{\sigma}}$ in finite time, $e \rightarrow 0$ as $t \rightarrow \infty$, and $\delta \tilde{a}_{2 j i}, \delta \tilde{a}_{1 j i}, \delta \tilde{b}_{2 j i}$, and $\delta \tilde{b}_{1 j i}$ remain bounded.

Remark 3.2. Since the control signal (3.4) contains the discontinuous sign functions, as a hard switcher, it may cause undesirable chattering. In order to avoid the chattering, the "sgn" function can be replaced by a continuous function (tanh) to remove discontinuity. 


\section{Numerical Simulation}

In this section, we present numerical results to verify the proposed synchronization approach. Consider the following master chaotic system:

$$
\begin{aligned}
{\left[\begin{array}{l}
\dot{x}_{1} \\
\dot{x}_{2} \\
\dot{x}_{3}
\end{array}\right]=} & {\left[\begin{array}{c}
a\left(x_{2}-x_{1}\right) \\
b x_{1}-c x_{1} x_{3} \\
-g x_{3}+h x_{1}^{2}
\end{array}\right]=\left[\begin{array}{ccc}
-a & a & 0 \\
b & 0 & 0 \\
0 & 0 & -g
\end{array}\right]\left[\begin{array}{l}
x_{1} \\
x_{2} \\
x_{3}
\end{array}\right]+\left[\begin{array}{ccc}
0 & 0 & 0 \\
0 & -c & 0 \\
0 & 0 & h
\end{array}\right]\left[\begin{array}{c}
0 \\
x_{1} x_{3} \\
x_{1}^{2}
\end{array}\right] } \\
= & \underbrace{\left[\begin{array}{ccc}
-a_{0} & a_{0} & 0 \\
b_{0} & 0 & 0 \\
0 & 0 & -g_{0}
\end{array}\right]}_{A_{1}} \underbrace{\left[\begin{array}{c}
x_{1} \\
x_{2} \\
x_{2}
\end{array}\right]}_{x}+\underbrace{\left[\begin{array}{ccc}
-\delta a_{0} & \delta a_{0} & 0 \\
\delta b_{0} & 0 & 0 \\
0 & 0 & -\delta g_{0}
\end{array}\right]}_{\Delta A_{1}} \underbrace{\left[\begin{array}{c}
x_{1} \\
x_{2} \\
x_{3}
\end{array}\right]}_{x} \\
& +\underbrace{\left[\begin{array}{ccc}
0 & 0 & 0 \\
0 & -c_{0} & 0 \\
0 & 0 & h_{0}
\end{array}\right]}_{B_{1}} \underbrace{\left[\begin{array}{c}
0 \\
x_{1} x_{3} \\
x_{1}^{2}
\end{array}\right]}_{f_{1}(x)}+\underbrace{\left[\begin{array}{ccc}
0 & 0 & 0 \\
0 & -\delta c_{0} & 0 \\
0 & 0 & \delta h_{0}
\end{array}\right]}_{\Delta B_{1}} \underbrace{\left[\begin{array}{c}
0 \\
x_{1} x_{3} \\
x_{1}^{2}
\end{array}\right]}_{f_{1}(x)},
\end{aligned}
$$

where $a=a_{0}+\delta a_{0}, b=b_{0}+\delta b_{0}, c=c_{0}+\delta c_{0}, g=g_{0}+\delta g_{0}, h=h_{0}+h g_{0}, a_{0}=8, \delta a_{0}=2, b_{0}=35$, $\delta b_{0}=5, c_{0}=0.7, \delta c_{0}=0.3, g_{0}=2.0, \delta g_{0}=0.5, h_{0}=0.8$, and $\delta h_{0}=0.2$.

The slave system is given with

$$
\begin{aligned}
{\left[\begin{array}{l}
\dot{y}_{1} \\
\dot{y}_{2} \\
\dot{y}_{3}
\end{array}\right]=} & {\left[\begin{array}{c}
a_{1}\left(y_{2}-y_{1}+y_{2} y_{3}\right) \\
b_{1} y_{2}-c_{1} y_{1} y_{3} \\
g_{1} y_{2}-h_{1} y_{3}
\end{array}\right]=\left[\begin{array}{ccc}
-a_{1} & a_{1} & 0 \\
0 & b_{1} & 0 \\
0 & g_{1} & -h_{1}
\end{array}\right]\left[\begin{array}{l}
y_{1} \\
y_{2} \\
y_{3}
\end{array}\right]+\left[\begin{array}{ccc}
a_{1} & 0 & 0 \\
0 & -c_{1} & 0 \\
0 & 0 & 0
\end{array}\right]\left[\begin{array}{c}
y_{2} y_{3} \\
y_{1} y_{3} \\
0
\end{array}\right] } \\
= & \underbrace{\left[\begin{array}{ccc}
-a_{10} & a_{10} & 0 \\
0 & b_{10} & 0 \\
0 & g_{10} & -h_{10}
\end{array}\right]}_{A_{2}} \underbrace{\left[\begin{array}{c}
y_{1} \\
y_{2} \\
y_{3}
\end{array}\right]}_{y}+\underbrace{\left[\begin{array}{ccc}
-\delta a_{10} & \delta a_{10} & 0 \\
0 & \delta b_{10} & 0 \\
0 & \delta g_{10} & -\delta h_{10}
\end{array}\right]}_{\Delta A_{2}} \underbrace{\left[\begin{array}{c}
y_{1} \\
y_{2} \\
y_{3}
\end{array}\right]}_{y} \\
& +\underbrace{\left[\begin{array}{ccc}
a_{10} & 0 & 0 \\
0 & -c_{10} & 0 \\
0 & 0 & 0
\end{array}\right]}_{B_{2}} \underbrace{\left[\begin{array}{c}
y_{2} y_{3} \\
y_{1} y_{3} \\
0
\end{array}\right]}_{f_{2}(y)}+\underbrace{\left[\begin{array}{ccc}
\delta a_{10} & 0 & 0 \\
0 & -\delta c_{10} & 0 \\
0 & 0 & 0
\end{array}\right]}_{\Delta B_{2}} \underbrace{\left[\begin{array}{c}
y_{2} y_{3} \\
y_{1} y_{3} \\
0
\end{array}\right]}_{f_{2}(y)}+\left[\begin{array}{c}
u_{1} \\
u_{2} \\
u_{3}
\end{array}\right],
\end{aligned}
$$

where $a_{1}=a_{10}+\delta a_{10}, b_{1}=b_{10}+\delta b_{10}, c_{1}=c_{10}+\delta c_{10}, g_{1}=g_{10}+\delta g_{10}, h_{1}=h_{10}+h g_{10}, a_{10}=0.8$, $\delta a_{10}=0.2, b_{10}=2.0, \delta b_{10}=0.5, c_{10}=0.7, \delta c_{10}=0.3, g_{10}=0.7, \delta g_{10}=0.3, h_{10}=3.0, \delta h_{10}=1.0$. $x(0)=1.8, y(0)=-1.2$, and $z(0)=1.5$.

The initial states in master system $(4.1)$ are $x_{1}(0)=1.8, x_{2}(0)=-1.2, x_{3}(0)=1.5$. The initial states in slave system $(4.2)$ are $y_{1}(0)=1.5, y_{2}(0)=1.2, y_{3}(0)=1$.1. The initial parameter estimation values of the systems (2.1) and (2.2) are $\delta \widehat{a}_{0}=0, \delta \widehat{b}_{0}=0, \delta \widehat{c}_{0}=0, \delta \widehat{g}_{0}=0, \delta \widehat{h}_{0}=0$, $\delta \widehat{a}_{10}=0, \delta \widehat{b}_{10}=0, \delta \widehat{c}_{10}=0, \delta \widehat{g}_{10}=0$, and $\delta \widehat{h}_{10}=0$. 


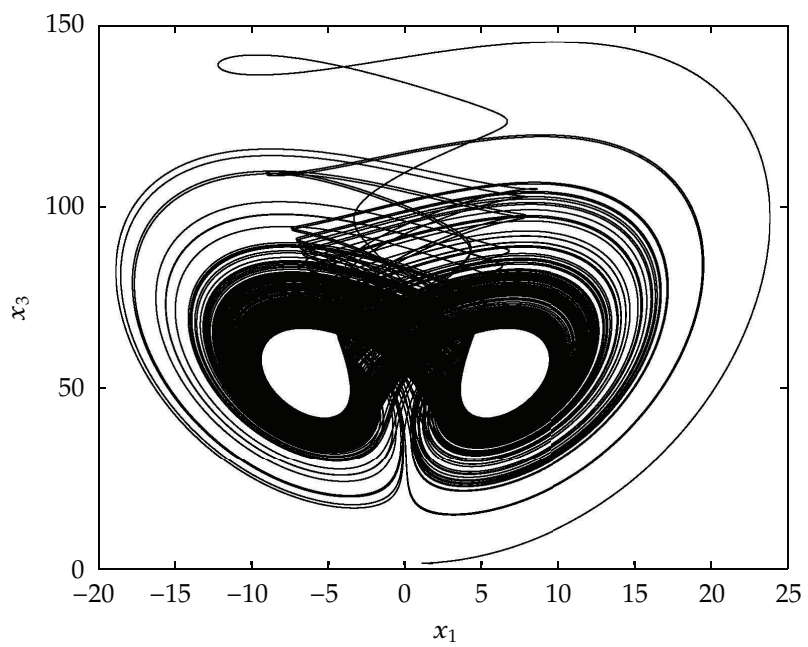

Figure 1: Chaotic behavior of the master chaotic system under the proposed parameters.

According to Remark 3.2, the control law (3.4) is modified as follows:

$$
\begin{aligned}
u=- & K e-K_{D} \dot{e}+\alpha_{s}-C_{A 2}-C_{B 2}+C_{A 1}+C_{B 1} \\
-\mu\left[2\left(\left|\delta \widehat{a}_{0}\right|+d_{a 0}\right)^{1+\alpha}+\left(\left|\delta \widehat{b}_{0}\right|+d_{b 0}\right)^{1+\alpha}+\left(\left|\delta \widehat{c}_{0}\right|+d_{c 0}\right)^{1+\alpha}\right. & \\
& +\left(\left|\delta \widehat{g}_{0}\right|+d_{g 0}\right)^{1+\alpha}+\left(\left|\delta \widehat{h}_{0}\right|+d_{h 0}\right)^{1+\alpha}+3\left(\left|\delta \widehat{a}_{10}\right|+d_{a 10}\right)^{1+\alpha}+\left(\left|\delta \widehat{b}_{10}\right|+d_{b 10}\right)^{1+\alpha} \\
& \left.+\left(\left|\delta \widehat{c}_{10}\right|+d_{c 10}\right)^{1+\alpha}+\left(\left|\delta \widehat{g}_{10}\right|+d_{g 10}\right)^{1+\alpha}+\left(\left|\delta \widehat{h}_{10}\right|+d_{h 10}\right)^{1+\alpha}\right] \alpha_{e}
\end{aligned}
$$

$-\left[c_{1} \tanh \left(\varepsilon e_{1}\right)\left|e_{1}\right|^{\alpha}, \ldots, c_{n} \tanh \left(\varepsilon e_{n}\right)\left|e_{n}\right|^{\alpha}\right]^{T}$,

where

$$
\begin{gathered}
C_{A 2}=\left[\delta \widehat{a}_{10}\left(y_{2}-y_{1}\right), \delta \widehat{b}_{10} y_{2}, \delta \widehat{g}_{10} y_{2}-\delta \widehat{h}_{10} y_{3}\right]^{T}, \\
C_{B 2}=\left[\delta \widehat{a}_{10} y_{2} y_{3},-\delta \widehat{c}_{10} y_{1} y_{3}, 0\right]^{T}, \quad C_{A 1}=\left[\delta \widehat{a}_{0}\left(x_{2}-x_{1}\right), \delta \widehat{b}_{0} x_{1},-\delta \widehat{g}_{0} x_{3}\right]^{T}, \\
C_{B 1}=\left[0,-\delta \widehat{c}_{0} x_{1} x_{3}, \delta \widehat{h}_{0} x_{1}^{2}\right]^{T}, \quad K=\operatorname{diag}\{70,54,30\}, \\
K_{D}=\operatorname{diag}\{0.93,0.75,0.1\}, \quad \mu=\operatorname{diag}\{1,0.2,0.01\}, \\
K_{A}=\operatorname{diag}\{2.3,2.1,2.3\} d_{a 0}=2, \quad d_{b 0}=10, \quad d_{c 0}=1, \quad d_{g 0}=1, \quad d_{h 0}=2, \\
d_{a 10}=1, \quad d_{b 10}=2, \quad d_{c 10}=2, \quad d_{g 10}=2, \quad d_{h 10}=2, \quad \varepsilon=40, \quad c_{1}=1, \quad c_{2}=3, \quad c_{3}=1 .
\end{gathered}
$$




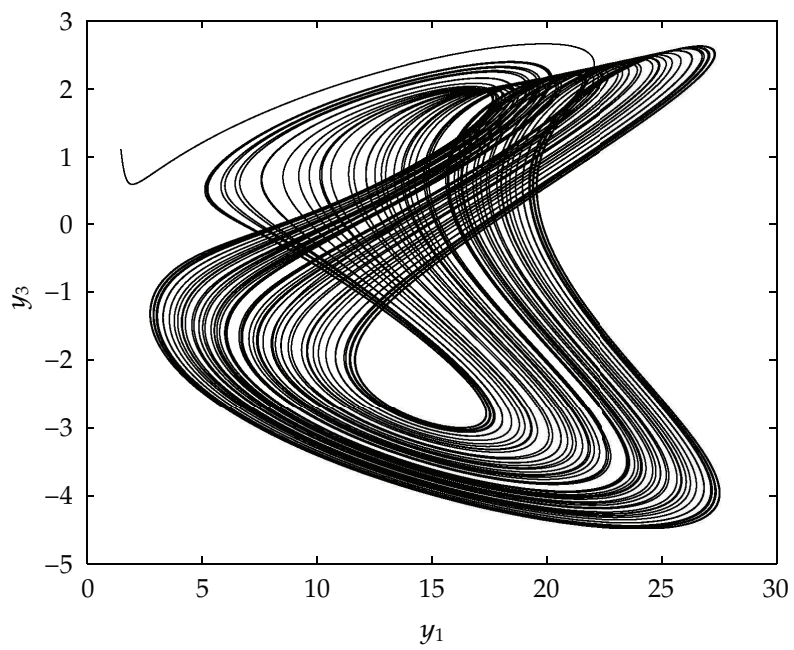

Figure 2: Chaotic behavior of the slave chaotic system under the proposed parameters.

Choosing the updating law as

$$
\begin{aligned}
& \delta \dot{\vec{a}}_{0}=\left\{\begin{array}{ll}
0.30 e_{1}\left(x_{1}-x_{2}\right), & \text { if }\left|\widehat{a}_{0}\right|<d_{a 0} \\
0, & \text { otherwise }
\end{array}, \quad \delta \hat{\vec{b}}_{0}= \begin{cases}-0.90 e_{2} x_{1}, & \text { if }\left|\widehat{b}_{0}\right|<d_{b 0} \\
0, & \text { otherwise }\end{cases} \right. \\
& \delta \dot{\hat{c}_{0}}=\left\{\begin{array}{ll}
-0.001 e_{2} x_{1} x_{3}, & \text { if }\left|\widehat{c}_{0}\right|<d_{c 0} \\
0, & \text { otherwise }
\end{array}, \quad \delta \dot{\hat{g}}_{0}= \begin{cases}0.002 e_{3} x_{3}, & \text { if }\left|\widehat{g}_{0}\right|<d_{g 0} \\
0, & \text { otherwise }\end{cases} \right. \\
& \delta \dot{\hat{h}_{0}}=\left\{\begin{array}{ll}
-0.018 e_{3} x_{1}^{2}, & \text { if }\left|\widehat{h}_{0}\right|<d_{h 0} \\
0, & \text { otherwise }
\end{array}, \quad \delta \dot{\hat{a}}_{10}= \begin{cases}0.0006 e_{1}\left(-y_{1}+y_{2}+y_{2} y_{3}\right), & \text { if }\left|\widehat{a}_{10}\right|<d_{a 10} \\
0, & \text { otherwise }\end{cases} \right. \\
& \delta \dot{\vec{b}}_{10}=\left\{\begin{array}{ll}
-0.08 e_{2} y_{2}, & \text { if }\left|\widehat{b}_{10}\right|<d_{b 10} \\
0, & \text { otherwise }
\end{array}, \quad \delta \dot{\hat{c}}_{10}= \begin{cases}-0.0015 e_{2} y_{1} y_{3}, & \text { if }\left|\widehat{c}_{10}\right|<d_{c 10} \\
0, & \text { otherwise }\end{cases} \right. \\
& \delta \dot{\hat{g}}_{0}=\left\{\begin{array}{ll}
0.1 e_{3} y_{2}, & \text { if }\left|\widehat{g}_{10}\right|<d_{g 10} \\
0, & \text { otherwise }
\end{array}, \quad \delta \dot{\hat{h}}_{10}= \begin{cases}-0.017 e_{3} y_{3}, & \text { if }\left|\widehat{h}_{10}\right|<d_{h 10} \\
0, & \text { otherwise. }\end{cases} \right.
\end{aligned}
$$

Chaotic behavior of the master chaotic system under the proposed parameters is shown in Figure 1. Chaotic behavior of the slave chaotic system under the proposed parameters is shown in Figure 2. From Figures 1 and 2, we know that the two systems are still chaotic under adopted uncertain parameters. The synchronization errors between two different chaotic systems are illustrated in Figures 3, 4, and 5, where the control inputs are activated at $t=1 \mathrm{~s}$. One can see that the synchronization errors converge to the zero in a finite time, which implies that the chaos synchronization between the two different chaotic systems 


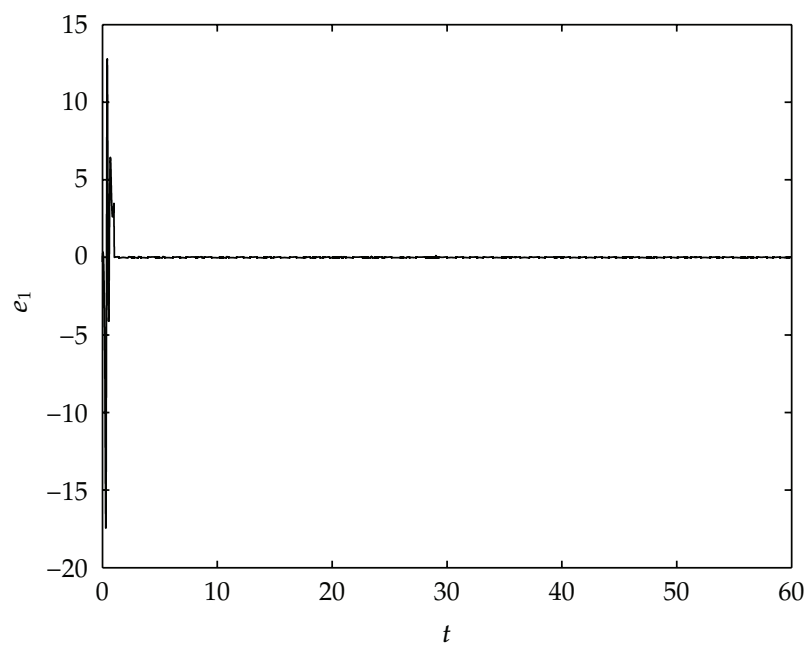

Figure 3: Synchronization error $e_{1}$.

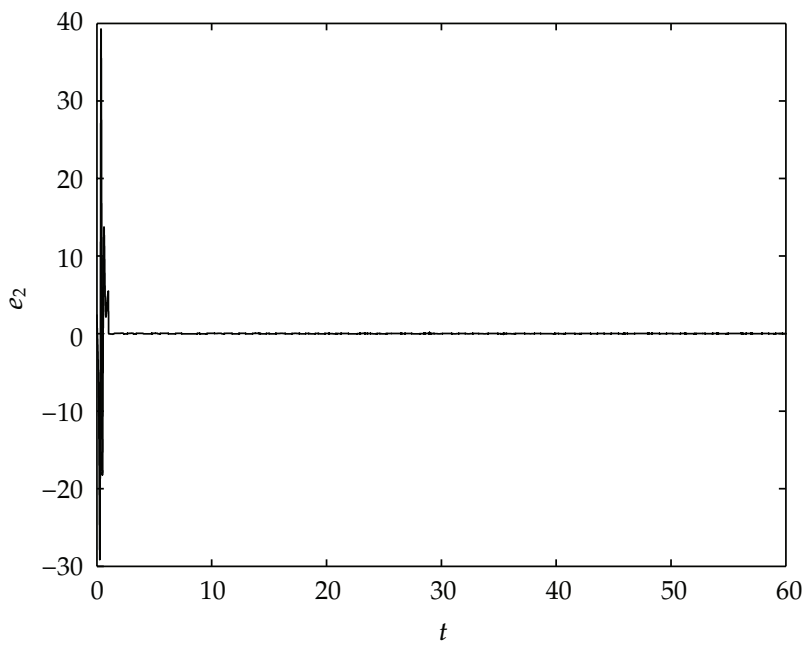

Figure 4: Synchronization error $e_{2}$.

is realized. The time responses of parameter estimations $\widehat{a}_{0}, \widehat{b}_{0}, \widehat{c}_{0}, \widehat{g}_{0}$, and $\widehat{h}_{0}$ are depicted in Figure 6. The time responses of parameter estimations $\widehat{a}_{10}, \widehat{b}_{10}, \widehat{c}_{10}, \widehat{g}_{10}$, and $\widehat{h}_{10}$ are depicted in Figure 7.

According to the simulations, it has been shown that the proposed control algorithm provides stable behavior when using online adaptive laws. The control performance is satisfactory and the chattering phenomenon has been successfully improved by using tanh functions. In addition, it is easy to see that the parameter estimation values approach their real values in a finite time. 


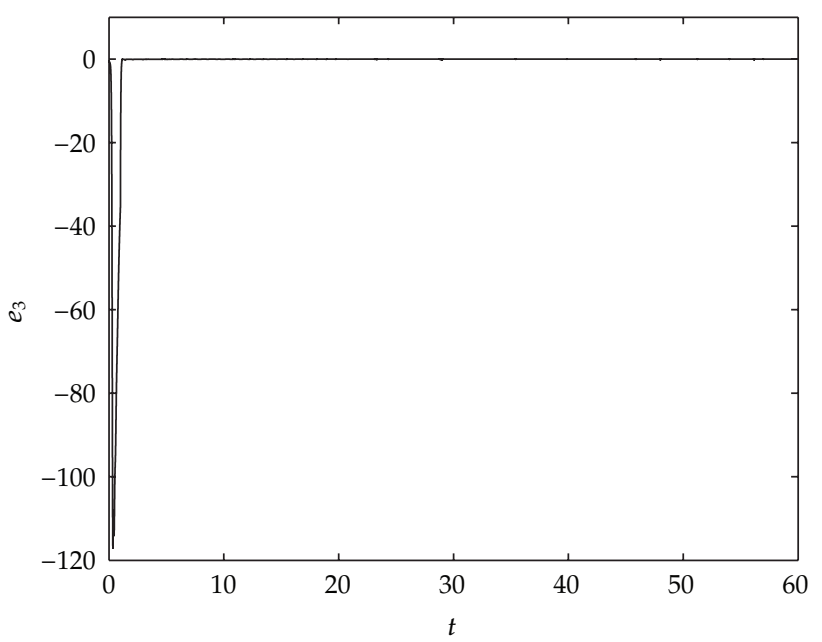

Figure 5: Synchronization error $e_{3}$.

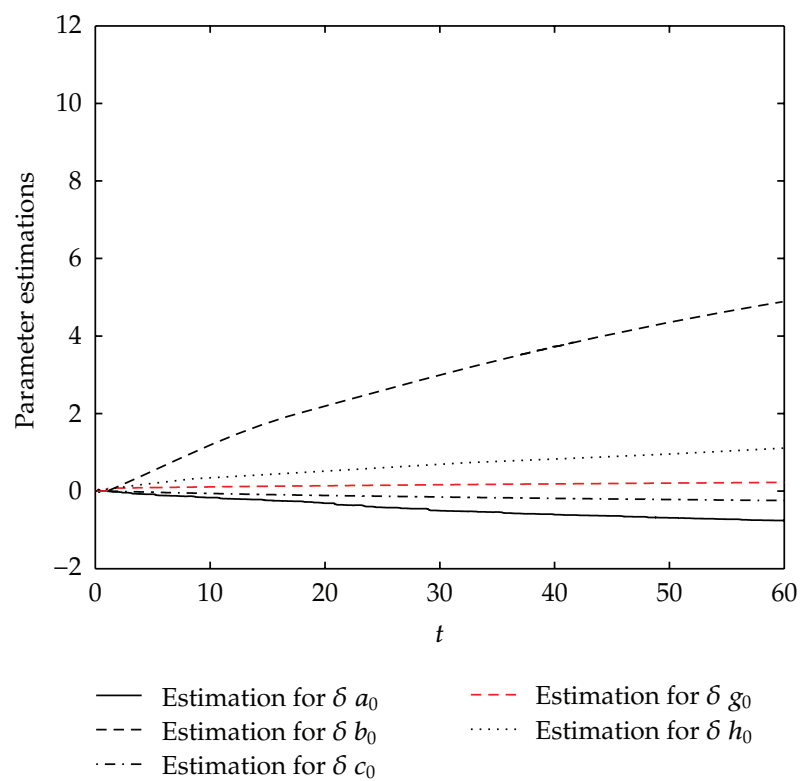

Figure 6: Curves of parameter estimations for the master system.

\section{Conclusions}

In this paper, we have studied chaos synchronization of two different chaotic systems with parameter uncertainties. The two different chaotic systems with parameter uncertainties are synchronized via robust adaptive control based on the Lyapunov stability theory and finitetime theory. The proposed method can be applied to a variety of chaos systems. It guarantees that all the error states are driven to zero in a finite time. Numerical simulations are given to show the proposed synchronization approach works well for synchronizing two different 


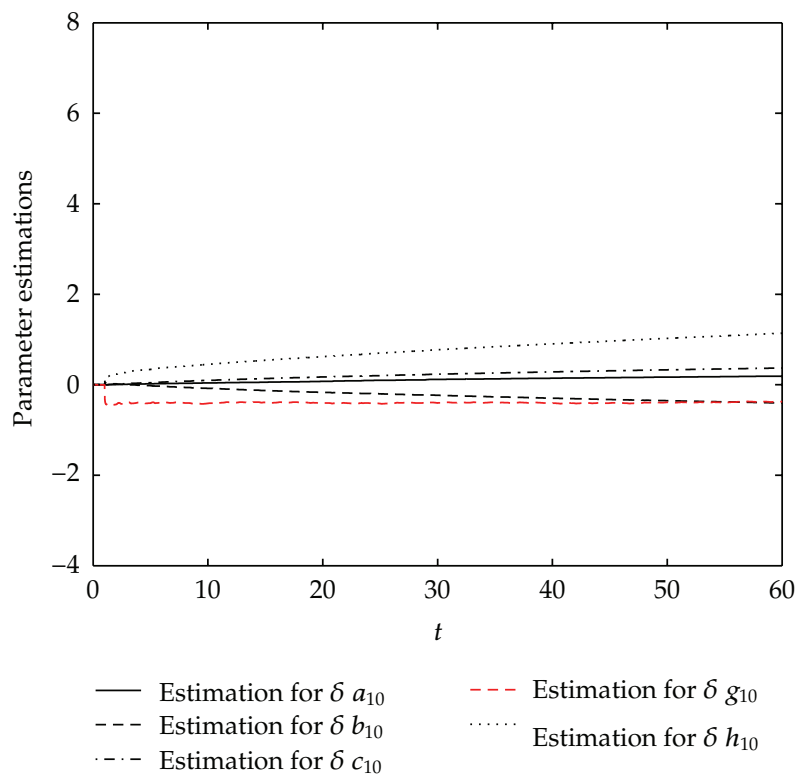

Figure 7: Curves of parameter estimations for the slave system.

chaotic systems in a finite time, even when the parameters of both the master and slave systems are unknown.

\section{Acknowledgment}

This work was supported by the National Natural Science Foundation of China under Grant no. 60674090 .

\section{References}

[1] G. Chen, Controlling Chaos and Bifurcations in Engineering Systems, CRC Press, Boca Raton, Fla, USA, 2000.

[2] S. Boccaletti, J. Kurths, G. Osipov, D. L. Valladares, and C. S. Zhou, "The synchronization of chaotic systems," Physics Reports, vol. 366, no. 1-2, pp. 1-101, 2002.

[3] Z. Yan, "Controlling hyperchaos in the new hyperchaotic Chen system," Applied Mathematics and Computation, vol. 168, no. 2, pp. 1239-1250, 2005.

[4] L. M. Pecora and T. L. Carroll, "Synchronization in chaotic systems," Physical Review Letters, vol. 64, no. 8, pp. 821-824, 1990.

[5] F. Wang and C. Liu, "A new criterion for chaos and hyperchaos synchronization using linear feedback control," Physics Letters, Section A, vol. 360, no. 2, pp. 274-278, 2006.

[6] X. Wu and H. Zhang, "Synchronization of two hyperchaotic systems via adaptive control," Chaos, Solitons and Fractals, vol. 39, no. 5, pp. 2268-2273, 2009.

[7] H. Y. Li and Y. A. Hu, "Robust sliding-mode backstepping design for synchronization control of crossstrict feedback hyperchaotic systems with unmatched uncertainties," Communications in Nonlinear Science and Numerical Simulation, vol. 16, no. 10, pp. 3904-3913, 2011.

[8] J. Wang, J. Gao, and X. Ma, "Synchronization control of cross-strict feedback hyperchaotic system based on cross active backstepping design," Physics Letters, Section A, vol. 369, no. 5-6, pp. 452-457, 2007. 
[9] H. Zhang, X. K. Ma, M. Li, and J. L. Zou, "Controlling and tracking hyperchaotic Rossler system via active backstepping design," Chaos, Solitons and Fractals, vol. 26, no. 2, pp. 353-361, 2005.

[10] Y. Yu and S. Zhang, "Adaptive backstepping synchronization of uncertain chaotic system," Chaos, Solitons and Fractals, vol. 21, no. 3, pp. 643-649, 2004.

[11] C. Wang and S. S. Ge, "Synchronization of two uncertain chaotic systems via adaptive backstepping," International Journal of Bifurcation and Chaos in Applied Sciences and Engineering, vol. 11, no. 6, pp. 17431751, 2001.

[12] M. Pourmahmood, S. Khanmohammadi, and G. Alizadeh, "Synchronization of two different uncertain chaotic systems with unknown parameters using a robust adaptive sliding mode controller," Communications in Nonlinear Science and Numerical Simulation, vol. 16, no. 7, pp. 2853-2868, 2011.

[13] C. Zhu, "Adaptive synchronization of two novel different hyperchaotic systems with partly uncertain parameters," Applied Mathematics and Computation, vol. 215, no. 2, pp. 557-561, 2009.

[14] J. Ma, A.-H. Zhang, Y.-F. Xia, and L.-P. Zhang, “Optimize design of adaptive synchronization controllers and parameter observers in different hyperchaotic systems," Applied Mathematics and Computation, vol. 215, no. 9, pp. 3318-3326, 2010.

[15] X. Wang and M. Wang, "Adaptive robust synchronization for a class of different uncertain chaotic systems," International Journal of Modern Physics B, vol. 22, no. 23, pp. 4069-4082, 2008.

[16] X. Wu, Z.-H. Guan, and Z. Wu, "Adaptive synchronization between two different hyperchaotic systems," Nonlinear Analysis: Theory, Methods \& Applications, vol. 68, no. 5, pp. 1346-1351, 2008.

[17] S.-Y. Li and Z.-M. Ge, "Pragmatical adaptive synchronization of different orders chaotic systems with all uncertain parameters via nonlinear control," Nonlinear Dynamics, vol. 64, no. 1-2, pp. 77-87, 2011.

[18] J. Huang, "Adaptive synchronization between different hyperchaotic systems with fully uncertain parameters," Physics Letters, Section A, vol. 372, no. 27-28, pp. 4799-4804, 2008.

[19] J. J. Yan, M. L. Hung, T. Y. Chiang, and Y. S. Yang, "Robust synchronization of chaotic systems via adaptive sliding mode control," Physics Letters, Section A, vol. 356, no. 3, pp. 220-225, 2006.

[20] J. B. Guan, "Synchronization control of two different chaotic systems with known and unknown parameters," Chinese Physics Letters, vol. 27, no. 2, Article ID 020502, 2010.

[21] J. H. Park, "Chaos synchronization between two different chaotic dynamical systems," Chaos, Solitons and Fractals, vol. 27, no. 2, pp. 549-554, 2006.

[22] H.-H. Chen, G.-J. Sheu, Y.-L. Lin, and C.-S. Chen, “Chaos synchronization between two different chaotic systems via nonlinear feedback control," Nonlinear Analysis: Theory, Methods E Applications, vol. 70, no. 12, pp. 4393-4401, 2009.

[23] G. H. Li, S. P. Zhou, and K. Yang, "Generalized projective synchronization between two different chaotic systems using active backstepping control," Physics Letters, Section A, vol. 355, no. 4-5, pp. 326-330, 2006.

[24] B. A. Idowu, U. E. Vincent, and A. N. Njah, “Generalized adaptive backstepping synchronization for non-identical parametrically excited systems," Nonlinear Analysis: Modelling and Control, vol. 14, no. 2, pp. 165-176, 2009.

[25] W. J. Yoo, D. H. Ji, and S. C. Won, "Adaptive fuzzy synchronization of two different chaotic systems with stochastic unknown parameters," Modern Physics Letters B, vol. 24, no. 10, pp. 979-994, 2010.

[26] C.-L. Kuo, "Design of a fuzzy sliding-mode synchronization controller for two different chaos systems," Computers E Mathematics with Applications, vol. 61, no. 8, pp. 2090-2095, 2011.

[27] M. Roopaei and M. Zolghadri Jahromi, "Synchronization of two different chaotic systems using novel adaptive fuzzy sliding mode control," Chaos, vol. 18, no. 3, Article ID 033133, 2008.

[28] M. P. Aghababa, S. Khanmohammadi, and G. Alizadeh, "Finite-time synchronization of two different chaotic systems with unknown parameters via sliding mode technique," Applied Mathematical Modelling, vol. 35, no. 6, pp. 3080-3091, 2011.

[29] M. Yahyazadeh, A. Ranjbar Noei, and R. Ghaderi, "Synchronization of chaotic systems with known and unknown parameters using a modified active sliding mode control," ISA Transactions, vol. 50, no. 2, pp. 262-267, 2011.

[30] M. Haeri and A. A. Emadzadeh, "Synchronizing different chaotic systems using active sliding mode control," Chaos, Solitons and Fractals, vol. 31, no. 1, pp. 119-129, 2007.

[31] J. Huang, "Chaos synchronization between two novel different hyperchaotic systems with unknown parameters," Nonlinear Analysis: Theory, Methods E Applications, vol. 69, no. 11, pp. 4174-4181, 2008.

[32] H. Wang, Z.-Z. Han, Q.-Y. Xie, and W. Zhang, "Finite-time chaos control via nonsingular terminal sliding mode control," Communications in Nonlinear Science and Numerical Simulation, vol. 14, no. 6, pp. 2728-2733, 2009. 
[33] W. Yu, "Finite-time stabilization of three-dimensional chaotic systems based on CLF," Physics Letters A, vol. 374, no. 30, pp. 3021-3024, 2010.

[34] H. Wang, Z.-z. Han, Q.-y. Xie, and W. Zhang, "Finite-time synchronization of uncertain unified chaotic systems based on CLF," Nonlinear Analysis: Real World Applications, vol. 10, no. 5, pp. 2842-2849, 2009.

[35] H. Wang, Z. Z. Han, Q. Y. Xie, and W. Zhang, "Finite-time chaos synchronization of unified chaotic system with uncertain parameters," Communications in Nonlinear Science and Numerical Simulation, vol. 14, no. 5, pp. 2239-2247, 2009.

[36] N. Cai, W. Li, and Y. Jing, "Finite-time generalized synchronization of chaotic systems with different order," Nonlinear Dynamics, vol. 64, no. 4, pp. 385-393, 2011.

[37] K. J. Astrom and B. Wittenmark, Adaptive Control, Pearson Education, 1995. 


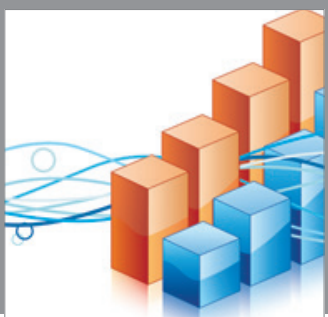

Advances in

Operations Research

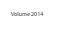

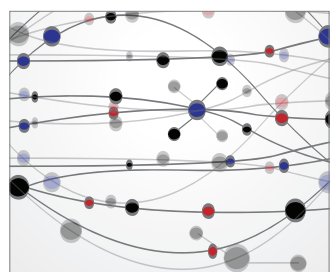

\section{The Scientific} World Journal
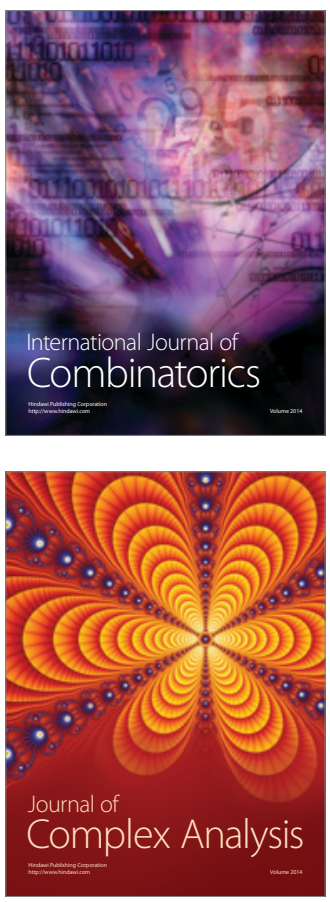

International Journal of

Mathematics and

Mathematical

Sciences
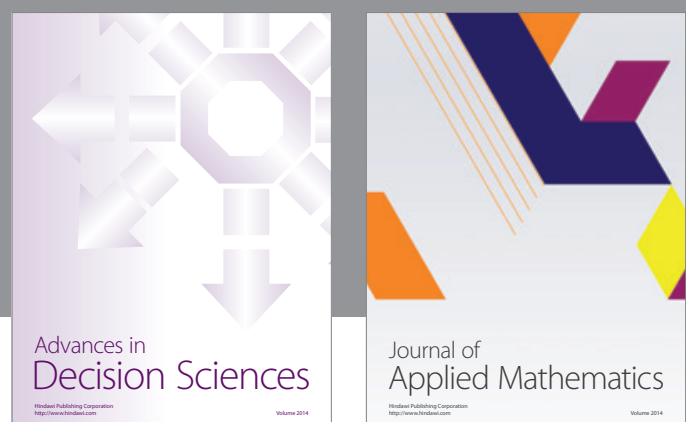

Journal of

Applied Mathematics
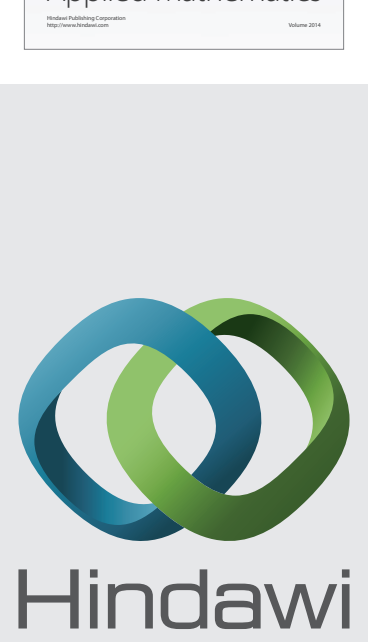

Submit your manuscripts at http://www.hindawi.com
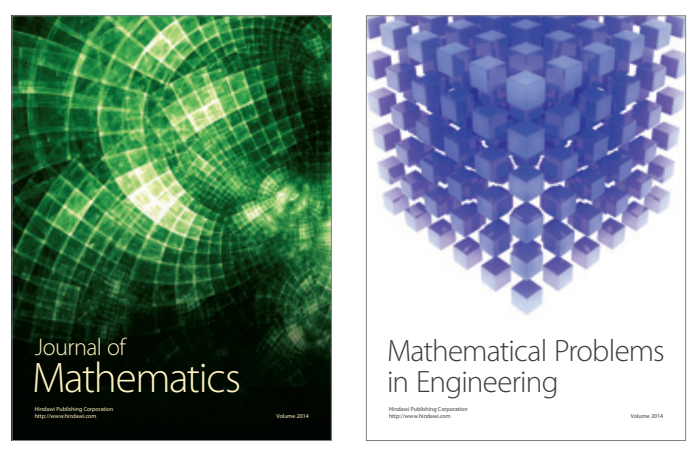

Mathematical Problems in Engineering
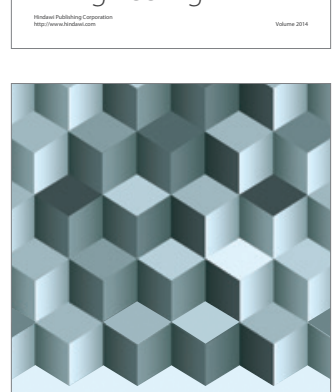

Journal of

Function Spaces
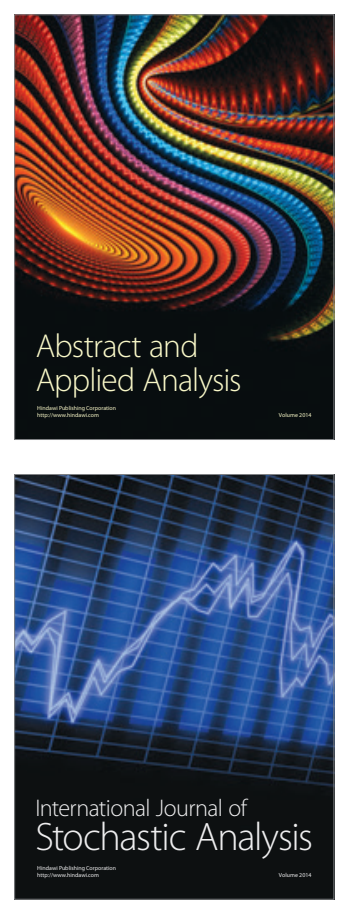

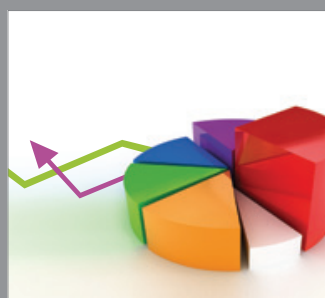

ournal of

Probability and Statistics

Promensencen
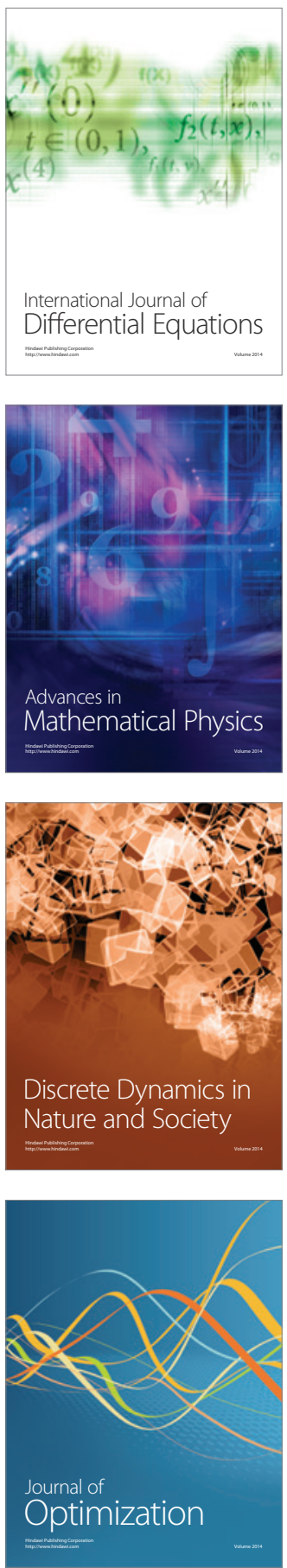